\title{
Conformations of individual quadruplex units studied in the context of extended human telomeric DNA $\uparrow$
}

\author{
Vijay Singh, $\ddagger$ Mykhailo Azarkh, Malte Drescher* and Jörg S. Hartig*
}

\begin{abstract}
G-quadruplex conformations within a sequence of three quadruplex units of human telomeric DNA were studied by two-frequency pulsed electron paramagnetic resonance (EPR) spectroscopy. In contrast to some individual G-quadruplexes, within the higher-order human telomeric sequence a $(3+1)$ hybrid structure is formed.
\end{abstract}

Telomeres are nucleic acid protein complexes located at the end regions of eukaryotic chromosomes and play important roles in maintaining the stability and integrity of chromosomes. ${ }^{1}$ The human telomeric DNA (HT DNA) consists of a duplex region composed of TTAGGG repeats with a $100200 \mathrm{nt}$ single stranded overhang of the $G$ rich strand at the $3^{\prime}$ end. $^{2}$ This overhang is a critical component of telomere structure and functions and has received a considerable amount of attention due to its structural complexity and biological role. For example, telomeric $\mathrm{G}$ quadruplexes have been extensively studied as a target for anticancer agents and are required for telomere end protection and extension. ${ }^{3}$

The structure and stability of $\mathrm{G}$ quadruplexes depend on the experimental conditions and the type of monovalent cations present. Most previous studies on telomeric $G$ quadruplexes were focused on single $\mathrm{G}$ quadruplexes units formed by four telomeric repeats. In $\mathrm{Na}^{+}$containing solution, an individual HT DNA quadruplex unit forms an antiparallel basket quadruplex topology. ${ }^{4}$ In the presence of $\mathrm{K}^{+}$in crystals a HT DNA quadruplex unit adopts a parallel structure also referred to as the propeller form (see Fig. 1). ${ }^{5}$ However in physiologically more relevant $\mathrm{K}^{+}$containing solutions there is a controversy regarding the quadruplex topology and many studies have reported the co existence of more than one quadruplex conformation. ${ }^{6}$ By utilizing Double Electron Electron Resonance (DEER) spectroscopy we have recently shown that an individual HT DNA quadruplex unit $\mathrm{d}\left[\mathrm{A}\left(\mathrm{G}_{3} \mathrm{~T}_{2} \mathrm{~A}\right)_{3} \mathrm{G}_{3}\right]$ adopts a $1: 1$ mixture of the parallel propeller and the antiparallel basket topology in $\mathrm{K}^{+}$solution. ${ }^{7}$ Using the virtually background free

Department of Chemistry and Konstanz Research School Chemical Biology, University of Konstanz, 78457 Konstanz, Germany.

Email: joerg.hartig@uni konstanz.de,malte.drescher@uni konstanz.de $\dagger$ Electronic supplementary information (ESI) available: Experimental details, synthetic procedure of spin labels, spin labeled oligonucleotide synthesis, CD spectra and EPR measurements. See DOI: 10.1039/ c2cc32012f

$\ddagger$ Present address: Department of Chemistry, Stanford University, Stanford, CA 94305, USA. a)

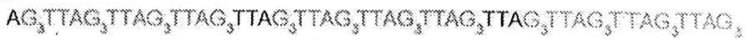

b)

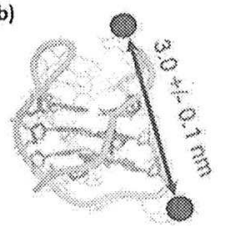

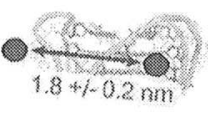

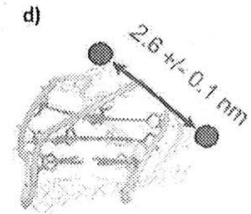

Fig. 1 (a) The HT DNA sequence $d\left[A\left(G_{3} T_{2} A\right)_{11} G_{3}\right]$ consists of three $\mathrm{G}$ quadruplex forming units depicted in red, green and orange, the connecting sequences (CS) linking individual quadruplex units are shown in black. (b) (d) Different intramolecular quadruplex structures of single quadruplex units based on high resolution structures. Arrows between blue circles indicate interspin distances measured between nitroxide spin labels. ${ }^{7}$ (b) NMR structure of the antiparallel basket quadruplex in $\mathrm{Na}^{+}$containing solution (PDB: 143D). ${ }^{4}$ (c) Crystal structure of the parallel propeller form in the presence of $\mathrm{K}^{+}$ions (PDB: $1 \mathrm{KF} 1) .^{5}$ (d) NMR structure of a $(3+1)$ hybrid quadruplex of a sequence slightly diverging from the human telomeric repeat in presence of $\mathrm{K}^{+}$(PDB: 2GKU) ${ }^{6}$ p

technique of in cell $\mathrm{EPR}^{8}$ we demonstrated that the same mixture of structures is formed when an unfolded HT DNA quadruplex unit was injected into Xenopus laevis oocytes. ${ }^{9}$

Although the structures of individual quadruplexes are well studied, there is an increasing need for characterizing the structure of extended sequences that better resemble the $3^{\prime}$ terminal, single stranded overhang of vertebrate telomeric DNA. Further insights into the structure of the full length overhang would be beneficial in order to shed light on questions such as the architecture and function of $\mathrm{t}$ and $\mathrm{D}$ loops and the interactions of the telomeric DNA with proteins and transcribed telomeric DNA (TERRA) in the shelterin complex. ${ }^{10}$

Until recently, only little was known about the structure of longer sequences of the telomere repeats overhang. ${ }^{11}$ Two main questions have been expressed with respect to such extended telomeric sequences: what is the exact conformation of individual quadruplex units in long sequences? And second: do individual quadruplex units interact? With respect to the latter question, two different models have been postulated: single quadruplexes could be positioned like "pearls on a string" with the TTA loops separating individual quadruplexes. On the other hand, in principle individual quadruplex units could interact, e.g. by stacking interactions. The latter conformation was 
referred to as a "stack of coins". Whereas the latter question of interactions between individual quadruplex units has been addressed in several reports, the folds of individual quadru plexes in this arrangement have been addressed rarely so far.

Sugimoto et al. ${ }^{\text {l } a}$ reported the existence of both parallel and antiparallel quadruplex topologies in $\mathrm{K}^{+}$solution for long HT DNA by using polyacrylamide gel electrophoresis (PAGE), CD and UV melting experiments. They have proposed a "pearls on a string" model having both parallel and antiparallel quadruplex topologies in the same structure. In contrast to this, Yang et al. ${ }^{11 b}$ studied the quadruplex conformation of short HT DNA in $\mathrm{K}^{+}$solution by NMR and proposed a hybrid type quadruplex topology with a compact stacking ("stack of coins") structure for long HT DNA. In another study, Fletcher et al. ${ }^{11 c}$ have also reported a similar hybrid quadruplex topology for longer HT DNA sequences showing a compact stacked structure by native PAGE and CD spectroscopy. The aforementioned hypothesis was validated by Komiyama and co workers ${ }^{11 d}$ by AFM, FRET and CD experiments and they postulated the formation of a higher order packing structure by consecutive formation of hybrid quadruplex topologies in which the interface between quadruplex units is stabilized by specific stacking interactions of the loop nucleotides. Computational studies including molecular dynamics, free energy calculations and principal components analysis carried out by Neidle and co workers ${ }^{11 e}$ exhibited the presence of a parallel stranded topology in higher order $G$ quadruplex dimers and tetramers with stacking interactions between $G$ quadruplex units. In a recent study, Zhou and co workers ${ }^{11 f}$ have revealed a slow transformation of a hybrid type quadruplex topology into a parallel type topology in $\mathrm{K}^{+}$ solution under a molecularly crowded environment using $\mathrm{CD}$ experiments. In their model, the parallel propeller quadruplex units were separated with minimal interaction. Molecular modelling suggested that sequences containing 12 repeats form higher order structures with three contiguous quadruplexes, most likely with mixed hybrid conformations. ${ }^{11 g}$

Until now no high resolution structural data have been available for sequences containing more than one quadruplex units, and indeed it is a difficult task to precisely study such sequences by using traditional NMR or crystallographic methods. In addition, techniques such as CD, UV, EMSA, etc. usually utilized for studying quadruplex conformations are hampered by the fact that the conformation of the whole extended oligonucleotide is investigated without the possibility to specifically assess the structure of one quadruplex within the background of other quadruplex units. However, EPR methods such as DEER ${ }^{12}$ have been developed recently in order to investigate $\mathrm{G}$ quadruplex topologies. Unlike other spectroscopic methods EPR only detects unpaired electron spins and does not detect any background signals from diamagnetic materials. Hence it is a very useful technique for studying individual quadruplex conformations within the context of long HT DNA sequences. Here, we report quadruplex conformational study of long HT DNA d $\left[A\left(G_{3} T_{2} A\right)_{11} G_{3}\right]$ containing three quadruplex units. We have used a site specific double nitroxide spin labeling techni que for studying the topologies of individual quadruplex units. We found that both the middle and the $5^{\prime}$ terminal quadruplex unit adopt a $(3+1)$ hybrid structure in $\mathrm{K}^{+}$containing solutions.
In order to study the middle quadruplex unit of long HT DNA, TEMPO nitroxide spin labels were attached at positions 29 and 35 of the sequence $\mathrm{d}\left[\mathrm{A}\left(\mathrm{G}_{3} \mathrm{~T}_{2} \mathrm{~A}\right)_{11} \mathrm{G}_{3}\right]$ via reported phosphoramidite based chemistry (see ESI $\dagger){ }^{7}$ The CD spectra of long spin labeled HT DNA were very similar compared to the CD spectra of unmodified DNA both in $\mathrm{Na}^{+}$ or $\mathrm{K}^{+}$containing solutions, confirming that the spin labels did not alter the quadruplex structure (see Fig. SI, ESI $\dagger$ ). For DEER distance measurements, a double spin labeled sequence ( $75 \mu \mathrm{M}$ in Tris $\mathrm{HCl}, \mathrm{pH} 7.5$ ) was annealed in the presence of $100 \mathrm{mM} \mathrm{NaCl}$ or $\mathrm{KCl}$. The annealed conformations were trapped by shock freezing in liquid nitrogen and the measure ments were carried out at $45 \mathrm{~K}$. Data were analysed using DEER Analysis 2010. ${ }^{13} \mathrm{~A}$ model free Tikhonov regularization analysis ${ }^{14}$ revealed that the distance distributions of the spin labels can be well characterized by Gaussian like distributions. The distance constraints indicated correspond to the centers of these Gaussians. Measurements on singly labeled sequences excluded intermolecular quadruplex formation (Fig. S3, ESI $\dagger$ ).

In the presence of $\mathrm{Na}^{+}$ions, the distance constraint between two nitroxide spin labels in the middle quadruplex unit of long HT DNA was found to be $3.0 \pm 0.1 \mathrm{~nm}$ (see Fig. 2a and b), which corresponds to the antiparallel basket quadruplex topology (see Fig. 1b). The presence of this quadruplex topology in $\mathrm{Na}^{+}$ solution is also supported by the respective $\mathrm{CD}$ spectra with a maximum at $295 \mathrm{~nm}$ and a minimum at $265 \mathrm{~nm}$, confirming the presence of the antiparallel topology (see Fig. S1b, ESI $\dagger$ ). However in $\mathrm{K}^{+}$solution the distance constraint of long HT DNA was measured to be $2.5 \pm 0.1 \mathrm{~nm}$ (see Fig. $2 \mathrm{c}$ and d). This indicates the presence of a $(3+1)$ hybrid topology (see Fig. 1d). This finding is in contrast to measurements we have carried out with a shorter four repeat DNA containing an individual HT DNA quadruplex unit $d\left[A\left(G_{3} T_{2} A\right)_{3} G_{3}\right]$. For the individual quadruplex unit we found a $1: 1$ mixture of parallel and antiparallel quadruplex topologies formed in $\mathrm{K}^{+}$solution. ${ }^{7}$ Hence it seems that the embedding of a single quadruplex unit into the longer sequence context containing more HT DNA repeats results in an alternative conformation. a)

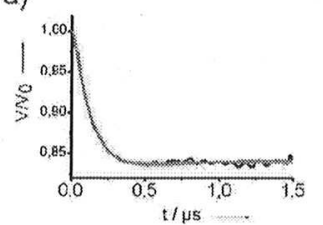

c)

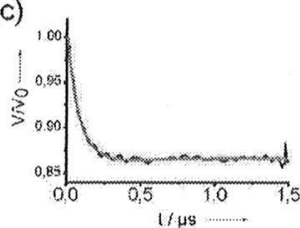

b)

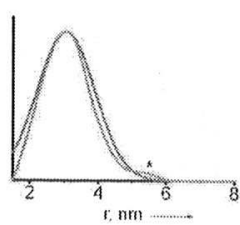

d)

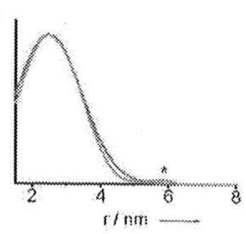

Fig. 2 DEER spectroscopy data for the middle $\mathrm{G}$ quadruplex unit of the HT DNA sequence containing three quadruplex units. Dipolar evolution after background correction in the presence of $\mathrm{Na}^{+}$(a) and $\mathrm{K}^{+}$(c) solutions with fit from Tikhonov regularization (cyan) and Gaussian model (red). Corresponding distance distributions are shown for $\mathrm{Na}^{+}$(b) and in $\mathrm{K}^{+}$containing (d) solutions. Asterisks mark artefacts due to Tikhonov regularization. 
a)

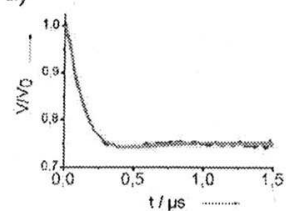

c)

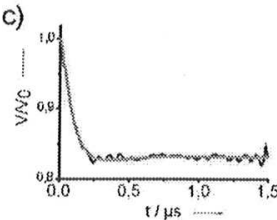

b)

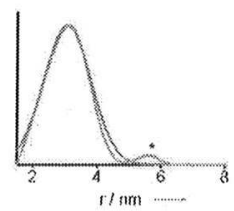

d)

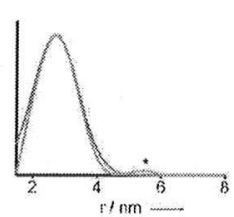

Fig. 3 DEER spectroscopic data probing the $5^{\prime}$ terminal quadruplex unit of the HT DNA sequence containing three quadruplex units. Dipolar evolution after background correction in the presence of $\mathrm{Na}^{+}$ (a) and $\mathrm{K}^{+}$(c) solutions with fit from Tikhonov regularisation (cyan) and Gaussian model (red). Corresponding distance distributions in $\mathrm{Na}^{+}(\mathrm{b})$ and in $\mathrm{K}^{+}$(d) containing solutions. Asterisks mark artefacts due to Tikhonov regularization.

This finding is supported by high resolution NMR studies showing such hybrid topologies when the HT core sequence is flanked by additional nucleotides. ${ }^{6 p, q, 11 h}$

We next determined a terminal quadruplex conformation by focusing on the $5^{\prime}$ quadruplex unit within the long triple unit HT DNA sequence. For this purpose, positions 5 and 11 of the sequence $d\left[A\left(G_{3} T_{2} A\right)_{11} G_{3}\right]$ were modified with the TEMPO label. Similar to the results obtained for the middle quadruplex unit, the quadruplex conformation of the $5^{\prime}$ terminal quadru plex in $\mathrm{Na}^{+}$solution also corresponded to the basket structure with a distance constraint of $3.0 \pm 0.1 \mathrm{~nm}$ (see Fig. 3a and b). In the presence of $\mathrm{K}^{+}$ions the distance constraint of the terminal quadruplex unit was found to be $2.7 \pm 0.1 \mathrm{~nm}$ (see Fig. $3 c$ and $d)$, indicating the existence of the $(3+1)$ hybrid structure (see Fig. 1d). By using site directed spin labeling techniques we found that in contrast to short HT DNA $\mathrm{d}\left[\mathrm{A}\left(\mathrm{G}_{3} \mathrm{~T}_{2} \mathrm{~A}\right)_{3} \mathrm{G}_{3}\right]$ both $5^{\prime}$ terminal and middle quadruplex units of the long HT DNA d $\left[A\left(G_{3} T_{2} A\right)_{11} G_{3}\right]$ adopt $(3+1)$ hybrid conformations in $\mathrm{K}^{+}$ion containing solution.

In conclusion, by using DEER spectroscopy we found that in contrast to short HT DNA, in extended sequences of HT DNA that contain three quadruplex forming units quadru plexes adopt a $(3+1)$ hybrid structure in $\mathrm{K}^{+}$containing solutions. This is in agreement with former reports that show the formation of hybrid structures for short but slightly extended quadruplexes in contrast to blunt ended quadruplex sequences. ${ }^{6 p, q}$ The present work is the first to show the utilization of EPR spectroscopy for investigating the confor mation of an individual quadruplex within a set of quadruplex forming units. This background free technique should prove to be very useful for further investigations of quadruplex structures and interactions in complex mixtures or even cellular systems.

JSH thanks the VolkswagenStiftung for funding of a Lichtenberg Professorship. Support of the Zukunftskolleg and the Deutsche Forschungsgemeinschaft (DR 743/2 1) is gratefully acknowledged.

\section{Notes and references}

1 (a) E. H. Blackburn, Nature, 1991, 350, 569; (b) V. A. Zakian, Science, 1995, 270, 1601; (c) C. W. Greider, Annu. Rev. Biochem., 1996, 65, 337.

2 (a) R. K. Moyzis, J. M. Buckingham, L. S. Cram, M. Dani, L. L. Deaven, M. D. Jones, J. Meyne, R. L. Ratliff and J. R. Wu, Proc. Natl. Acad. Sci. U. S. A., 1988, 85, 6622; (b) V. L. Makarov, Y. Hirose and J. P. Langmore, Cell, 1997, 88, 657.

3 (a) S. Neidle and G. Parkinson, Nat. Rev. Drug Discovery, 2002, 1, 383 (b) L. H. Hurley, Nat. Rev. Cancer, 2002, 2, 188; (c) S. Neidle, Curr. Opin. Struct. Biol., 2009, 19, 239; (d) S. Balasubramanian and S. Neidle, Curr. Opin. Chem. Biol., 2009, 13, 345; (e) E. H. Blackburn, Cell, 2001, 106, 661; (f) T. R. Cech, Cell, 2004, 116, 273; (g) J. Karlseder and R. E. Verdun, Nature, 2007, 447, 924; $(h)$ T. de Lange, Nat. Rev. Mol. Cell Biol., 2004, 5, 323; (j) W. E. Wright, V. M. Tesmer, K. E. Huffman, S. D. Levene and J. W. Shay, Genes Dev., 1997, 11, 2801; (k) N. Maizels, Nat. Struct. Mol. Biol., 2006, 13, 1055.

4 Y. Wang and D. J. Patel, Structure, 1993, 1, 263.

5 G. N. Parkinson, M. P. Lee and S. Neidle, Nature, 2002, 417, 876.

6 (a) J. Dai, M. Carver and D. Yang, Biochimie, 2008, 90, 1172; (b) A. Ambrus, D. Chen, J. Dai, T. Bialis, R. A. Jones and D. Yang, Nucleic Acids Res., 2006, 34, 2723; (c) P. Balagurumoorthy and S. K. Brahmachari, J. Biol. Chem., 1994, 269, 21858; (d) Y. He, R. D. Neumann and I. G. Panyutin, Nucleic Acids Res., 2004, 32, 5359; (e) L. Ying, J. J. Green, H. Li, D. Klenerman and S. Balasubramanian, Proc. Natl. Acad. Sci. U. S. A , 2003, 100, 14629; (f) A. Risitano and K. R. Fox, Bioorg. Med. Chem. Lett., 2005, 15, 2047; (g) I. N. Rujan, J. C. Meleney and P. H. Bolton, Nucleic Acids Res, 2005, 33, 2022; (h) I. Ourliac Garnier, M. A. Elizondo Riojas, S. Redon, N. P. Farrell and S. Bombard, Biochemistry, 2005, 44, 10620; (i) A. Wlo darczyk, P. Grzybowski, A. Patkowski and A. Dobek, J. Phys. Chem. B, 2005, 109, 3594; (j) J. Qi and R. H. Shafer, Nucleic Acids Res., 2005, 33, 3185; (k) S. Redon, S. Bombard, M. A. Elizondo Riojas and J. C. Chottard, Nucleic Acids Res., 2003, 31, 1605; (l) J. Li, J. J. Correia, L. Wang, J. O. Trent and J. B. Chaires, Nucleic Acids Res., 2005, 33, 4649; (m) A. Risitano and K. R. Fox, Nucleic Acids Res., 2004, 32, 2598; (n) P. Hazel, J. Huppert, S. Balasubramanian and S. Neidle, J. Am. Chem. Soc., 2004, 126, 16405; (o) A. Benz and J. S. Hartig, Chem. Commun., 2008, 4010; (p) K. N. Luu, A. T. Phan, V. Kuryavyi, L. Lacroix and D. J. Patel, J. Am. Chem. Soc., 2006, 128, 9963; (q) D. J. E. Yue, K. W. Lim and A. T. Phan, J. Am. Chem. Soc., $2011,133,11462$

7 V. Singh, M. Azarkh, T. E. Exner, J. S. Hartig and M. Drescher, Angew. Chem., Int. Ed., 2009, 48, 9728.

8 (a) M. Azarkh, O. Okle, V. Singh, I. T. Seemann, J. S. Hartig D. R. Dietrich and M. Drescher, ChemBioChem, 2011, 12, 1992; (b) I. Krstić, R. Hansel, O. Romainczyk, J. W. Engels, V. Dotsch and T. F. Prisner, Angew. Chem., Int. Ed., 2011, 50, 5070.

9 M. Azarkh, V. Singh, O. Okle, D. R. Dietrich, J. S. Hartig and M. Drescher, ChemPhysChem, 2012, 13, 1444.

10 L. E. Donate and M. A. Blasco, Philos. Trans. R. Soc., B, 2011, 366, 76

11 (a) H. Q. Yu, D. Miyoshi and N. Sugimoto, J. Am. Chem. Soc. 2006, 128, 15461; (b) A. Ambrus, D. Chen, J. Dai, T. Bialis, R. A. Jones and D. Yang, Nucleic Acids Res., 2006, 34, 2723; (c) I. M. Pedroso, L. F. Duarte, G. Yanez, K. Burkewitz and T. M. Fletcher, Biopolymers, 2007, 87, 74; (d) Y. Xu, T. Ishizuka, K. Kurabayashi and M. Komiyama, Angew. Chem., Int. Ed., 2009, 48, 7833; (e) S. Haider, G. N. Parkinson and S. Neidle, Biophys. J., 2008, 95, 296; (f) L. Xu, S. Fenga and X. Zhou, Chem. Commun. 2011, 47, 3517; $(g)$ L. Petraccone, C. Spink, J. O. Trent, N. C. Garbett, C. S. Mekmaysy, C. Giancola and J. B. Chaires, J. Am. Chem. Soc., 2011, 133, 20951; (h) M. Vorlickova J. Chladkova, I. Kejnovska, M. Fialova and J. Kypr, Nucleic Acids Res., 2005, 33, 5851.

12 (a) A. D. Milov, A. B. Ponomarev and Y. D. Tsvetkov, Chem Phys. Lett., 1984, 110, 67; (b) M. Pannier, S. Veit, A. Godt G. Jeschke and H. W. Spiess, J. Magn. Reson., 2000, 142, 331 (c) G. Jeschke, ChemPhysChem, 2002, 3, 927.

13 G. Jeschke, V. Chechik, P. Ionita, A. Godt, H. Zimmermann, J. Banham, C. R. Timmel, D. Hilger and H. Jung, Appl. Magn Reson., 2006, 30, 473

14 Y. M. Chiang, P. P. Borbat and J. H. Freed, J. Magn. Reson., 2005, $172,279$. 